\title{
INSTITUTO DE ESTUDOS BRASILEIROS DA USP
}

Ao planejar e propor a fundação do Instituto de Estudos Brasileiros (IEB), criado pela Universidade de São Paulo em 1962, Sérgio Buarque de Holanda pretendia estabelecer na Universidade um centro interdisciplinar de pesquisa e documentação sobre a história e a cultura do país. Para tanto, destacava, entre seus principais objetivos: 1) propiciar o desenvolvimento de trabalhos conjuntos de professores e pesquisadores que, espalhados por várias unidades da USP, estudam, cada um em sua área, aspectos da cultura brasileira; 2) abrigar estudiosos que desenvolvem pesquisas permanentes e de longa duração; 3) ser capaz de colher, preservar e organizar fontes primárias para os estudos brasileiros, colocando-as a serviço da pesquisa. O IEB permanece fiel a estes objetivos iniciais que motivaram sua criação.

Seu Conselho Deliberativo é constituído por professores das Unidades da USP ligados às áreas voltadas para a cultura brasileira. No início, chamado Conselho Diretor, era formado por titulares de algumas disciplinas; depois, professores eleitos nos Departamentos da Faculdade de Filosofia, Letras e Ciências Humanas

A AUTORA
Marta Rossette Batista
Diretora do Instituto de Estudos Brasileiros,
IEB-USP

(FFLCH), Faculdade de Arquitetura e Urbanismo (FAU), Faculdade de Economia e Administração (FEA) e Faculdade de Educação (FE). Atualmente, com os novos Regimentos da USP e do IEB, no qual está definido como Instituto Especializado, órgão de integração, apoiando o ensino, a pesquisa e a extensão universitária. Seu Conselho Deliberativo compõe-se de conselheiros vindos de seis unidades afins, escolhidos por suas Congregações, ao lado dos conselheiros escolhidos dentre os integrantes da própria Instituição. São hoje definidas como instituições afins do IEB: Escola de Comunicações e Artes (ECA), FAU, Faculdade de Direito (FD), FEA, FE e FFLCH.

$\mathrm{O}$ IEB sempre se dedicou à pesquisa de longa duração. Desde o início contou, em caráter permanente, com pesquisadores da própria instituição, todos em tempo integral, a partir de 1968/69, desenvolvendo trabalhos individuais e interdisciplinares que marcaram o Instituto como centro de referência em áreas e linhas de pesquisa, tais como os estudos sobre a imigração, o Modernismo brasileiro e as edições críticas. $\mathrm{O}$ estudo acurado dos acervos propiciou o desenvolvimento de métodos e técnicas de pesquisa e de campos pouco explorados no Brasil, tais como: arquivologia, paleografia, documentação museológica e colecionismo, o estudo de periódicos e obras raras, edições críticas e fidedignas e, mais recentemente, 
manuscritologia e crítica genética. Hoje, integrando as áreas de História, Literatura, Artes Plásticas e Música, os docentes/pesquisadores do Instituto trabalham ainda no apoio ao ensino e à extensão universitária, transmitindo o conhecimento gerado em cursos no IEB e em outras Unidades da USP. No Instituto, deve-se destacar o Curso de Especialização em Organização de Arquivos, que, em seus 11 anos de existência, capacitou profissionais do país e no exterior.

O IEB também se mostrou capaz de reunir, organizar e colocar à disposição dos estudiosos documentos únicos e de destaque para a compreensão da cultura brasileira, formando um acervo expressivo e reconhecido.

À época da criação do Instituto, a USP adquiriu para o IEB a Brasiliana do historiador Yan de Almeida Prado, com 10 mil volumes, englobando obras raras ou de difícil acesso da época colonial, álbuns de viajantes, revistas brasileiras dos séculos XIX e $\mathrm{XX}$. A estas preciosidades acrescentaram-se as da Brasiliana do historiador Alberto Lamengo, transferida da FFLCH em 1968, com manuscritos da época colonial e contando com o livro mais antigo existente na USP, a Crônica de Nuremberg, de 1493. Destacaram-se, também, as aquisições de acervos de escritores, entre eles os de Mário de Andrade (1968), Guimarães Rosa (1973) e Graciliano Ramos (1980 e 1994), com seus arquivos pessoais, ricos em anotações e manuscritos, recortes e fotografias. Com o prestígio da atuação inicial do IEB, além de historiadores e escritores, outros intelectuais ou seus descendentes doaram à Instituição seus acervos pessoais. Estão entre eles a coleção do educador Fernando de Azevedo (1970), do poeta e crítico Theon
Spanudis (1987), da artista plástica Anita Malfatti (1989), do geógrafo Pierre Monbeig (1990), perfazendo, em 1996, 26 acervos a que se somam, através de doações ou compras, pequenas coleções, obras avulsas e material resultante de pesquisa. Podese estimar o Acervo-IEB em cerca de 110 mil volumes, 250 mil documentos e duas mil obras de arte.

Os acervos determinam tanto as linhas de pesquisa da Instituição quanto a própria organização interna dedicada a seu processamento. Nos primeiros anos, a Biblioteca abrigava as Brasilianas e seu estudo levou, entre outros, a uma grande atenção com as obras raras. Com a chegada do acervo Mário de Andrade, que incluía biblioteca de 17 mil volumes, arquivo pessoal com cerca de 30 mil documentos e um conjunto importante de mais de mil objetos e obras de arte, iniciou-se a definição, ainda embrionária, dos órgãos internos - que atualmente cuidam dos documentos e das obras, de arte - ao mesmo tempo em que as pesquisas sobre o Modernismo ganhavam impulso.

As obras de arte que haviam pertencido a Mário de Andrade, às quais foram se acrescentando as de vários escritores, passaram a ser estudadas e classificadas pelos pesquisadores. O IEB foi das primeiras instituições brasileiras a conservar este tipo de arquivos pessoais. Os trabalhos e arquivos pessoais se avolumaram, termimando por constituir outra seção interna dedicado a eles, o Arquivo, que mantém coleções diversificadas, muitas geradas por pesquisas específicas e doadas ao IEB.

Na Biblioteca do Instituto particular atenção é dada às obras raras e de luxo, às coleções de periódicos nacionais, e aos livros com dedicatória ou anotados por seus antigos proprietários - o que os torna 
também peças únicas. Na Coleção de Artes Visuais, a catalogação museológica procura avançar na documentação (muitas vezes existente em outras partes dos acervos, na Biblioteca ou no Arquivo), no estudo do artista ou artesão e na relação objeto/colecionador. No Arquivo, os inventários de cada série e subsérie detalham a origem do documento e sua relação com o produtor/coletor, numa revisão contínua dos métodos de classificação.

Em 1993, o IEB-USP mudou-se para uma sede de $2.800 \mathrm{~m}^{2}$ dos quais $1.800 \mathrm{~m}^{2}$ dedicados ao Acervo (armazenamento, processamento, consulta e exposição). Tem-se acentuado a preocupação com a preservação dos documentos e obras de arte, incluindo a preparação de áreas em que estão armazenados (hoje climatizadas e protegidas contra roubo e incêndio); os modos de armazenamento e acondicionamento e o preparo para consulta. Tornou-se possível um melhor atendimento, com a renovação de áreas e equipamentos de consulta e reprodução.

Esta preocupação do IEB com o trato dos acervos foi reconhecida pelo IPHAN, Instituto do Patrimônio Histórico e Artístico Nacional, que lhe concedeu o Prêmio Rodrigo Melo Franco de Andrade, na categoria Preservação de acervos culturais móveis e imóveis.

Com a aprovação de novo Regimento, em 1995, o IEB-USP desenvolveu e consolidou a estrutura, definida anteriormente, em torno do binômio pesquisa/acervos. A divisão de pesquisa $\mathrm{CaC}$, Câmara Científica, reúne docentes, com objetivo de traçar diretrizes para a pesquisa, ensino e extensão. A CSA, Comissão de Serviços de Apoio, congrega os órgãos dedicados ao Acervo: Arquivo, Biblioteca e Coleção de Artes Visuais - ao lado do Serviço de Difusão Cultural e de representação docente, para desenvolver a política dos acervos, sua recepção, processamento e divulgação.

Deve-se acrescentar que os acervos e as pesquisas contam, para sua divulgação, com um Serviço de Difusão Cultural, encarregado de Cursos e Publicações. As publicações compreendem catálogos, livros e a Revista do Instituto de Estudos Brasileiros, fundada em 1966. Os cursos, em expansão, incluem os de extensão e o de Especialização em Organização de Arquivos. Desde 1995, o IEB oferece disciplinas em nível de graduação da USP e estuda a implantação de um programa de pósgraduação em Cultura e Civilização Brasileiras.

O Acervo do IEB tem crescido continuamente e pode-se caracterizá-lo como uma reunião de acervos particulares de personalidades que se dedicaram a estudar a história e a cultura brasileiras ou que, com sua produção, contribuíram para o desenvolvimento e/ou mudanças do panorama cultural de nossa terra. $\mathrm{O}$ conjunto destes acervos torna o Instituto um centro rico para a pesquisa nas áreas de História, Literatura, Artes Plásticas, Músicas, Sociologia, Geografia, Educação, com destaque para temas como: o período colonial e a documentação jesuítica; o período do Império e os viajantes; a cultura popular; o Modernismo brasileiro na literatura, artes plásticas e música.

Durante os 35 anos de existência do IEB, muitos foram os que colaboraram para o enriquecimento do acervo, com doações e estímulo para o trabalho de sua organização, exploração e divulgação. Na impossibilidade de nomeá-los, queremos agradecer a Antônio Cândido, José Aderaldo Castello e José Mindlin, tomados por nós como representação de todos. 


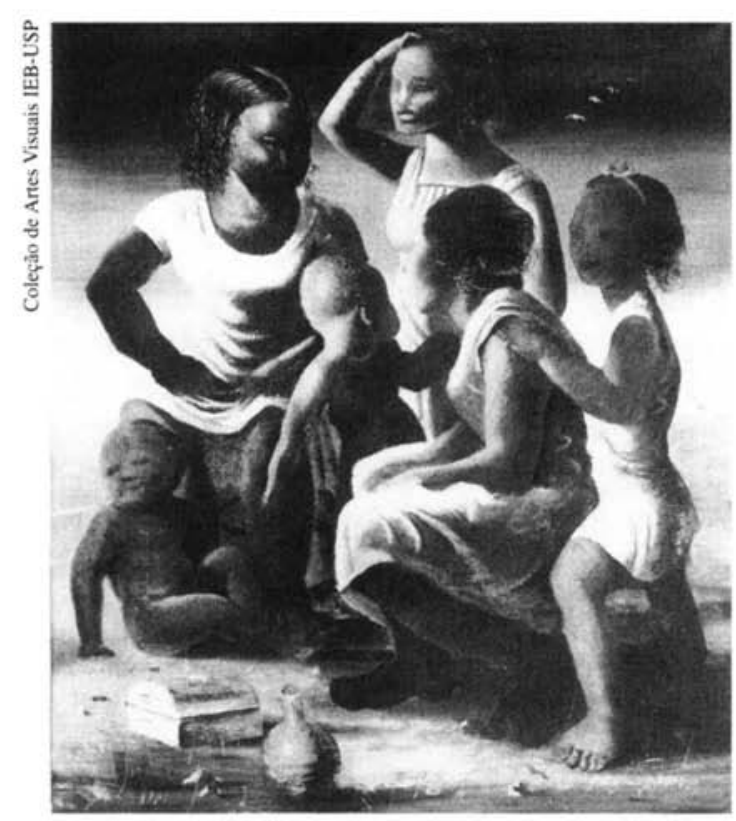

Cândido Portinari. Composição Retirantes, 1936. (óleo s/ tela $73.3 \times 59,5$ )

\section{ARQUIVO}

\section{Informações Gerais}

Horário de atendimento:

- de segunda à sexta-feira, das $9 \mathrm{~h}$ às $12 \mathrm{~h} \mathrm{e}$ das $13 \mathrm{~h}$ às $17 \mathrm{~h}$

Serviços oferecidos:

- atendimento a consulentes, visitantes, órgãos oficiais e culturais e imprensa

- orientação para uso do acervo

- estágio supervisionado para interessados em organização de acervo arquivístico

Público a que se destina:

- pesquisadores e estudiosos em geral

Orientação para uso do acervo:

O Consulente deve inscrever-se de acordo com o objetivo do trabalho. $\mathrm{O}$ acesso ao documento será feito mediante consulta aos instrumentos de pesquisa. O consulente será esclarecido pelos técnicos quanto ao manuseio adequado dos documentos, recebendo informações sobre as possibilidades de reprodução (microfilme, fotografia ou xe- rox), conforme o tipo de documento, seu estado de conservação e as cláusulas específicas de cada fundo.

Instrumentos de pesquisa:

Inventários, catálogos, índices, publicados ou datilografados, e bancos de dados.

Composição e características:

O Arquivo do IEB surgiu em 1968, integrado à Biblioteca. Com o crescimento da massa documental transformou-se em serviço independente, a partir de 1974, com o objetivo de receber, organizar, preservar e divulgar o acervo, visando oferecer fontes primárias para pesquisa. Compreende os setores: Atendimento e Consulta; Documentação textual e Documentação audiovisual. No tratamento da documentação distingue:

Arquivos pessoais: conjunto de documentos de caráter pessoal, científico, artístico, profissional ou técnico, produzidos e/ou reunidos por uma pessoa, em decorrência de suas atividades.

Coleções: conjunto de documentos de vários tipos e origens reunidos por uma pessoa ou instituição, em geral relacionados a um assunto ou a uma personalidade.

Documentação: conjunto de documentos de várias procedências e assuntos, agrupados em:

Resultante de pesquisas: documentação original e/ou reproduzida, proveniente de pesquisas realizadas no IEB ou aí depositadas. Resultante de exposição: documentação reproduzida e reunida para exposições realizadas pela Instituição.

Documentação avulsa: documentos textuais, sonoros e visuais provenientes de doações eventuais sobre os mais variados assuntos da cultura brasileira.

O Arquivo abriga ainda:

Arquivo Permanente do IEB: conjunto de documentos pertencentes ao fundo IEB, 
preservados em caráter definitivo, dado o seu valor histórico. (Não aberto à consulta.) Atividades Culturais do IEB: série formada por conjuntos documentais (textual, sonoro e visual), produzidos e acumulados pelos serviços da Instituição: Difusão Cultural, Arquivo, Biblioteca e Coleção de Artes Visuais, resultantes de exposições, palestras, cursos e publicações. (Aberto à consulta.)

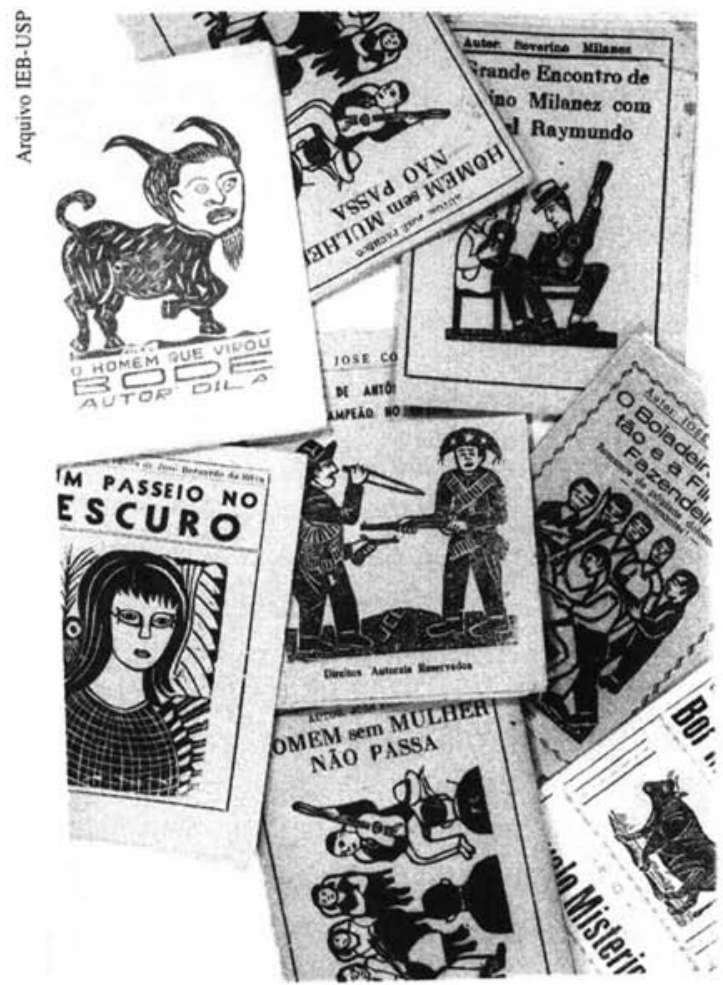

Coleção de Folhetos de Cordel

\section{BIBLIOTECA}

Informação gerais

Horário de atendimento:

- de segunda à sexta-feira, das $9 \mathrm{~h}$ às $17 \mathrm{~h}$

Serviços oferecidos:

- atendimento a usuários e visitantes

- orientação para o uso do acervo

- levantamento bibliográfico

- normalização bibliográfica
- comutação bibliográfica nacional e internacional

- treinamento informal dos usuários

- estágio para interessados em organização de acervo (bibliotecárias)

Público a que se destina:

- alunos de graduação e pós-graduação, professores, pesquisadores brasileiros e estrangeiros, público em geral interessado em cultura brasileira

Orientação para uso do acervo:

A consulta aos catálogos e fichários é livre e, em caso de necessidade, os usuários recebem orientação sobre seu uso. As obras solicitadas são consultadas no local, pois, devido às suas características peculiares, $o$ acervo não é de livre acesso e nem circulante, não havendo empréstimo entre bibliotecas. A Biblioteca orienta sobre a possibilidade de reprodução: é vedado fazer cópias (xerox) de obras em mau estado de conservação, manuscritos, primeiras edições, obras raras e obras com encadernações valiosas. Nestes casos, podem ser fotografadas ou microfilmadas, observadas as normas de funcionamento da Biblioteca e do IEB.

Instrumentos de pesquisa:

- catálogos de livros e periódicos

- DEDALUS: Bancos de Dados Bibliográficos da USP

- bases em CD ROM

Composição e características:

A Biblioteca do IEB é considerada hoje, qualitativamente, uma das mais ricas existentes em assuntos brasileiros e, quantitativamente, aproxima-se dos $110 \mathrm{mil}$ volumes. Seu ponto de partida foi a famosa Brasiliana do historiador paulista Yan de Almeida Prado comprada em 1962. Outras coleções, mantidas em sua unidade, portanto fixas e, em geral, com os nomes dos seus primeiros proprietários, foram sendo acrescentadas, por compra ou doação. São as seguintes: Coleções Mário de Andrade, 
Coleção Alberto Lamengo, Coleção Guerra do Paraguai, Coleção Juarez Bezerra de Menezes, Coleção Guimarães Rosa, Coleção José Feliciano de Oliveira, Coleção Alfredo Elis Júnior, Coleção Theon Spanudis, Coleção Ernani Silva Bruno, Coleção Pierre Monbeig, Coleção Raul de Andrada e Silva, Coleção Graciliano Ramos, Coleção Uacury Ribeiro de Assis. Ao lado dessas coleções, a Biblioteca possui sua Coleção Geral composta por pequenas doações, permutas e compras. Merece destaque especial o conjunto de revistas que a Biblioteca abriga. Possui 1.911 títulos de diferentes periódicos, em coleções nem sempre completas. A Biblioteca tem se empenhado em reunir revistas nacionais e estrangeiras, principalmente as revistas dos Institutos de Históricos e Geográficos dos Estados brasileiros e de centros latino-americanos e luso-brasileiros de diversos países. Entre as colações completas incluem-se: Antônio Maria, Astro da Lusitana, O Besouro, Cabrião, O Federalista, O Investigador Portuguez em Inglaterra, Klaxon, Kosmos, Lanterna Verde, O Polichinelo, Revista de Antropofagia, Verde etc., em suas edições originais.

\section{COLEÇÃO DE ARTES VISUAIS}

Informações Gerais

Horário de atendimento:

- de segunda à sexta-feira, das $9 \mathrm{~h}$ às $12 \mathrm{~h} \mathrm{e}$ das $14 \mathrm{~h}$ às $17 \mathrm{~h}$

- Exposição: aberta ao público de segunda à sexta-feira, das $14 \mathrm{~h}$ às $17 \mathrm{~h}$

Serviços oferecidos:

- atendimento a consulentes, visitantes, órgãos oficiais e culturais e imprensa

- orientação para o uso do acervo

- estágio supervisionado na área de museologia e arte brasileira (1917 - 1945)
- visita guiada à Exposição

Público a que se destina:

- pesquisadores, alunos de graduação e pós-graduação e estudiosos em geral do país e do exterior

- Exposição: público em geral

Orientação para o uso do acervo:

A visita às exposições é livre e gratuita

A documentação museológica pode ser consultada junto dos técnicos da Coleção, que informarão as condições para solicitação do exame das obras da reserva técnica, assim como para fotografar qualquer peça.

Instrumentos de pesquisa: Catálogos e listagens, fichas catalográficas das peças, documentação fotográfica, hemeroteca.

Composição e características:

A Coleção de Artes Visuais do IEB teve seu início com a chegada do Acervo Mário de Andrade, em 1968, composto de objetos e obras de arte que o escritor paulista colecionou. A Coleção Mário de Andrade, mantida em sua globalidade, permaneceu como museu de um único acervo, até 1981 quando, pelo novo Regimento do IEB, ampliou-se para Coleção de Artes Visuais, aberta a novas doações/aquisições de interesse para a arte brasileira.

A Coleção Mário de Andrade permanece como a mais importante e significativa e suas principais obras têm sido expostas de forma permanente desde a chegada do acervo ao IEB. No final dos anos 70 e sobretudo a partir de 1980 - com melhor espaço em nova sede - iniciaram-se as mostras periódicas, destacando artistas, temas ou pesquisas em torno da Coleção Mário de Andrade. A partir do final dos anos 80 também são apresentados, em periódicas, os novos acervos recebidos.

Desde o início, as obras recebem cuidados museológicos tradicionais, de identíficação, catalogação/documentação, conservação/restauro, pesquisa e divulgação em 
publicações e exposições, no IEB, na mídia e em significativas mostras de arte brasileira, no país e no exterior.

Os interessados em visitar o acervo do IEB-USP para realizar pesquisas ou mesmo para fazer consultas devem se dirigir ao seguinte endereço:

Av. Prof. Mello Moraes, 140 -

Travessa 8 - Cidade Universitária

05508-900 São Paulo - SP

Telefones:

Resumo: O Instituto de Estudos Brasileiros (IEB) da USP, fundado em 1962 por Sérgio Buarque de Holanda, é um centro interdisciplinar de pesquisa e documentação sobre a história e a cultura do país. O Arquivo, a Biblioteca e a Coleção de Artes Visuais congregam um acervo de 110 mil volumes, 250 mil documentos e 2 mil obras de arte que podem ser pesquisados e consultados por alunos, professores e pesquisadores.

Palavras-chave: Instituto de Estudos Brasileiros, cultura brasileira, biblioteca, arquivo, artes visuais
Arquivo: (011) 818-3427

Biblioteca: (011) 818-3467

Coleção de Artes Visuais:

(011) 818-3247

Difusão Cultural - Setor de Publicações:

(011) 818-3199

Setor de Cursos e Atividades Afins

(011) 818-3199

Fax (para todas as seções):

(011) 818-3143

E-mail: bibieb@brusp.bitnet

Abstract: USP's Instituto de Estudos Brasileiros (IEB, or Brazilian Studies Institute, BSI), founded in 1962 by Sérgio Buarque de Holanda, is an interdisciplinary center for research and documentation of the Country's history and culture. The Archives, Library and the Visual Art Collection congregate a collection of 110 thousand volumes, 250 thousand documents and two thousand works of art may be researched and consulted by students, professors and researchers.

Keywords: Instituto de Estudos Brasileiros, Brazilian culture, library, archive, visual art 